\title{
Engineered Mild Strains of Papaya ringspot virus for Broader Cross Protection in Cucurbits
}

\author{
Bang-Jau You, Chu-Hui Chiang, Li-Fang Chen, Wei-Chih Su, and Shyi-Dong Yeh
}

First, third, fourth, and fifth authors: Department of Plant Pathology, National Chung Hsing University, Taichung, Taiwan; and second author: Department of Molecular Biotechnology, Da Yeh University, Changhua, Taiwan.

Accepted for publication 16 December 2004.

\begin{abstract}
You, B.-J., Chiang, C.-H., Chen, L.-F., Su, W.-C., and Yeh, S.-D. 2005. Engineered mild strains of Papaya ringspot virus for broader cross protection in cucurbits. Phytopathology 95:533-540.

Papaya ringspot virus (PRSV) HA5-1, a mild mutant of type P Hawaii severe strain (PRSV P-HA), has been widely used for the control of PRSV type $\mathrm{P}$ strains in papaya, but did not provide practical protection against PRSV type $\mathrm{W}$ strains in cucurbits. In order to widen the protection effectiveness against $\mathrm{W}$ strains, chimeric mild strains were constructed from HA5-1 to carry the heterologous $3^{\prime}$ genomic region of a type W strain W-CI. Virus accumulation of recombinants and their cross-

horn melon and squash plants, the recombinant carrying both the heterologous coat protein $(\mathrm{CP})$ coding region and the $3^{\prime}$ untranslated region ( $3^{\prime} \mathrm{UTR}$ ), but not the heterologous CP coding region alone, significantly enhanced the protection against W-CI. The heterologous $3^{\prime} \mathrm{UTR}$ alone is critical for the enhancement of the protection against W-CI in horn melon, but not in zucchini squash. In papaya, the heterologous CP coding region or $3^{\prime} \mathrm{UTR}$ alone, but not both together, significantly reduced the effectiveness of cross protection against P-HA. Our recombinants provide broader protection against both type $\mathrm{W}$ and $\mathrm{P}$ strains in cucurbits; however, the protective effectiveness is also affected by virus accumulation, the organization of the $3^{\prime}$ genomic region, and host factors.
\end{abstract} protection effectiveness against W-CI and P-HA were investigated. In
Most strains of Papaya ringspot virus (PRSV), a member of the genus Potyvirus, belong to one of two major types (29). Type P isolates of PRSV (PRSV P, papaya-infecting) infect plants of $\mathrm{Cu}-$ curbitaceae, Chenopodiaceae, and Caricaceae and limit papaya plantation in tropical and subtropical areas. Type $\mathrm{W}$ isolates of PRSV (PRSV W, not papaya-infecting) infect plants of Cucurbitaceae and Chenopodiaceae and cause severe diseases in cucurbits worldwide. Type $\mathrm{P}$ and $\mathrm{W}$ isolates of PRSV are serologically indistinguishable; their major difference is papaya-infecting or not $(29,45)$.

Cross protection describes the phenomenon that plants systematically infected with one strain of a virus are protected from infection by a second related strain of the same virus (26). In practice, mild strains of Tobacco mosaic virus (TMV) (31), Citrus tristeza virus (7), PRSV (46), and Zucchini yellow mosaic virus $(22,39)$ have been used in large-scale application for control of the corresponding viruses. Among a group of virus strains, such as Tobacco streak virus (12), Sugarcane mosaic virus (21), and Barley yellow dwarf virus (41), some combinations show complete cross protection, whereas other combinations may exhibit only partial or no protection. The phenomenon of strain-specific protection is one of the major concerns for practical application of cross protection.

The mild PRSV strain HA5-1, which causes infection in papaya without conspicuous symptoms, was derived from a severe Hawaii PRSV type P strain (PRSV P-HA) by nitrous acid induction (43). HA5-1 provided high degrees of protection (90 to $100 \%$ ) against the severe parental strain P-HA under greenhouse and field conditions in Taiwan and Hawaii $(43,44,46)$. However, protection provided by HA5-1 against type $\mathrm{W}$ virus in

Corresponding author: S.-D. Yeh; E-mail address: sdyeh@nchu.edu.tw

DOI: 10.1094/PHYTO-95-0533

(c) 2005 The American Phytopathological Society horn melon only reached 20 to $30 \%$ (43). This strain-specific protection limits the application of HA5-1 for control of type W virus in cucurbits.

The blocking of disassembly of the challenge virus by the coat protein $(\mathrm{CP})$ was considered as a possible mechanism for cross protection $(10,35)$. Recently, the importance of the CP in cross protection was also demonstrated by use of a chimeric virus approach. Chimeric severe Potato virus $X$ (PVX) expressing the translatable TMV CP gene provides a higher degree of cross protection than PVX carrying nontranslatable TMV CP sequence (9). An amino acid substitution at the $\mathrm{N}$-terminal region of the $\mathrm{CP}$ was shown to affect cross-protection efficiency for chimeric Potato virus $A$ (PVA) between potato and tobacco strains (37). In addition, sequestration of the factor(s) or sites essential for the replication of the challenge virus by the protective strain was also proposed as a mechanism for cross protection (28). Therefore, the 3 '-terminal region of a potyviral genome encoding the $\mathrm{CP}$ reading frame and an untranslated region (UTR) essential for genomic amplification (11) may play important roles in cross protection.

Posttranscriptional gene silencing (PTGS) describes a phenomenon manifested by a constant transcription rate of a transgene in the nuclei of a transgenic plant, but an increased level of sequence-specific degradation of transgene mRNA in the cytoplasm $(23,36)$. Recently, many studies have indicated that PTGS is induced in plants infected with Nepovirus (32), Tobravirus (33), Caulimovirus (8), or Potexvirus (33). The small interfering RNA derived from viral RNA (17) acts as a guide for sequence-specific cleavage of the homologous RNA $(32,33)$. PTGS is considered an antiviral defense mechanism in virus-infected plants, playing an important role in cross protection (38). Therefore, a higher degree of sequence identity between a protective strain and a challenge strain may increase the cross-protection effectiveness.

In an effort to solve the problem of strain-specific cross protection of HA5-1 and widen the cross-protection effectiveness against type $\mathrm{W}$ strains in cucurbits, chimeric mild strains were constructed from HA5-1 to carry a heterologous CP coding region 
and/or a 3'UTR of a type W strain that originated from Taiwan (PRSV W-CI). Under greenhouse conditions, virus accumulation of recombinant mild strains and their effectiveness in cross protection against type $\mathrm{W}$ strain $\mathrm{W}-\mathrm{CI}$ and type $\mathrm{P}$ strain $\mathrm{P}-\mathrm{HA}$ in cucurbit and papaya plants were investigated. In cucurbits, the chimeric mild virus carrying the heterologous $\mathrm{CP}$ coding region and $3^{\prime} \mathrm{UTR}$ from W-CI provided a much higher degree of cross protection against W-CI than HA5-1, while still maintaining a high degree of cross protection against P-HA infection. Our results indicate that it is possible to confer broad protection against two distinct virus strains using a chimeric mild potyvirus carrying a heterologous $3^{\prime}$ genomic region. Also, cross-protection effectiveness of the recombinants is greatly affected by their accumulation in the host, the organization of the $3^{\prime}$ viral genomic region, and host factors.

\section{MATERIALS AND METHODS}

Virus sources. PRSV type P strain P-HA was isolated from papaya in Hawaii (14) and PRSV type W strain W-CI from Luffa gourd (Luffa cylindrica Roem) in Chiayi of Taiwan (40). The mild PRSV strain HA5-1 was derived from the severe Hawaii strain P-HA by nitrous acid induction (43). P-HA and HA5-1 were maintained in plants of papaya (Carica papaya L. var. Tainung No. 2), and W-CI was maintained in plants of horn melon ( $\mathrm{Cu}$ cumis metuliferus Naud [Mey. Acc. 2459]).
Construction of infectious clone of HA5-1. The in vitro fulllength infectious cDNA clone pT3-HAG of PRSV P-HA, driven by a bacteriophage T3 promoter, was previously constructed (6). Three cDNA clones (pM31, pW21, and pW29) corresponding to genome segments of $\mathrm{P} 1$ to $\mathrm{P} 3, \mathrm{P} 3$ to $\mathrm{CP}$, and nuclear inclusion $\mathrm{b}$ (NIb) to 3'UTR of PRSV HA5-1, respectively, were selected from a $\lambda$ ZAP II cDNA library (5) and used to replace the viral genome segment of pT3-HAG to create an in vitro infectious cDNA clone of HA5-1, pT3HA5-1 (Fig. 1A).

Construction of chimeric mild viruses. The cDNA fragment corresponding to the complete CP coding region and 3'UTR of W-CI was previously cloned (40). The chimeric construct pT3HA5-1Wcp3u was generated by replacing the ApaI-NotI fragment of pT3HA5-1, which contained the complete CP coding region, the 3'UTR, and 45 amino acids of the C-terminal region of the NIb coding region, with that of W-CI (Fig. 1A). The ApaINotI DNA fragment of pT3HA5-1 was inserted into pBluescriptII SK(-) and an SmaI site was generated 3 nucleotides from the termination codon of the $\mathrm{CP}$ coding region to create p5-1ANsmaI by site-directed mutagenesis (Gene Editor Kit; Promega, Madison, WI) with primer HASmaI (5'-AAACACACAAGCCCGGGTATTTAGTTGCGCATACCCAG-3'). The ApaI-NotI DNA fragment of pT3HA5-1 was replaced with the corresponding region of $\mathrm{p} 5-1 \mathrm{AN}$ smaI to create pT3HA5-1smaI. The entire $\mathrm{CP}$ coding region with a small C-terminal portion of the $\mathrm{NIb}$ coding region (45 amino acids) of W-CI was amplified by primers

A
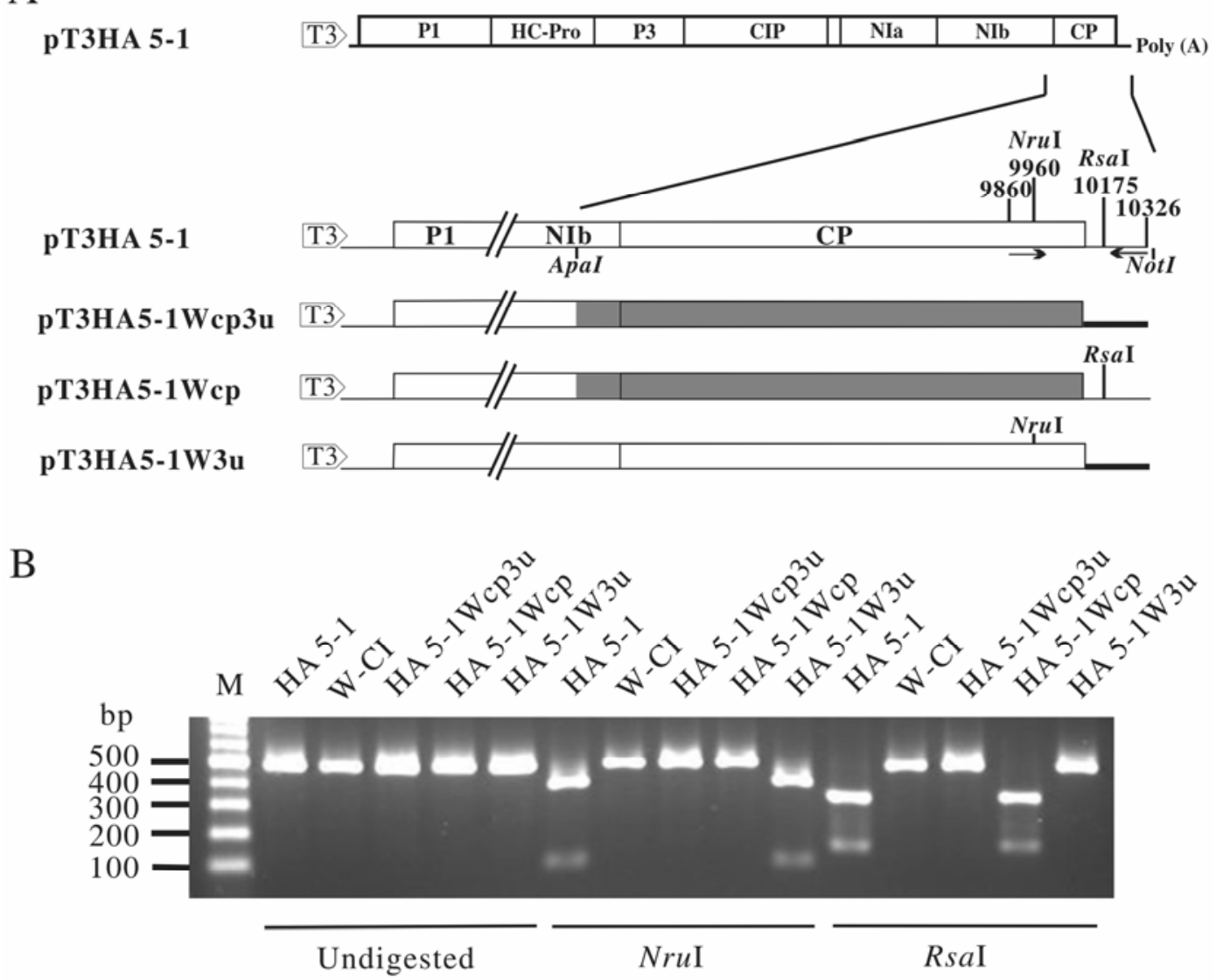

Fig. 1. Construction and verification of chimeric mild strains of Papaya ringspot virus (PRSV). A, Chimeric constructs pT3HA5-1Wcp3u, pT3HA5-1Wcp, and pT3HA5-1W3u based on the previously constructed in vitro infectious clone pT3HA5-1 of a type P mild strain of PRSV HA5-1. The fragment of pT3HA5-1 corresponding to the $\mathrm{C}$-terminal region of the nuclear inclusion $\mathrm{b}(\mathrm{NIb})$ gene ( 45 amino acids [aa]), the entire coat protein $(\mathrm{CP})$ coding region, and the complete $3^{\prime}$ untranslated region ( $3^{\prime} \mathrm{UTR}$ ) was replaced with the corresponding fragment of a type W strain W-CI of PRSV to generate the construct pT3HA5-1Wcp3u. The construct pT3HA5-1Wcp was derived from the replacement of the partial C-terminal region of NIb (45 aa) and the entire CP coding region of HA5-1 with the corresponding fragment from W-CI. The construct pT3HA5-1W3u was created by the replacement of the 3'UTR of pT3HA5-1 with that of W-CI. B, Confirmation of the fidelity of chimeric mild viruses. Total RNAs were extracted from plants of horn melon infected with HA5-1, W-CI, HA5-1Wcp3u, HA5-1Wcp, or HA51W3u. The targeted fragment of nucleotides 9860-10326 was amplified by reverse transcription-polymerase chain reaction with primers PW9860 and MW10326, digested with $N r u \mathrm{I}$ or $R s a \mathrm{I}$, and analyzed by agarose (1.2\%) gel electrophoresis. 
HASmaI (5'-AAACACACAAGCCCGGGTATTTAGTTGCGCATACCCAG-3') and YK905 (5'-GCAGGGCCCCATATGTGTCT$3^{\prime}$ ), digested with ApaI and SmaI, and inserted into ApaI/SmaIdigested pT3HA5-1smaI to create pT3HA5-1Wcp that contained a heterologous $\mathrm{CP}$ coding region (Fig. 1A). The cDNA fragment corresponding to the complete $3^{\prime} \mathrm{UTR}$ of W-CI was amplified by primers WcpSmaI (5'-TTGAATACCCGGGCTAGTG-3') and T7 (5'-GTAATACGACTCACTATAGGGC-3'), digested with SmaI and NotI, and then inserted into SmaI/NotI-digested p5-1ANsmaI to create plasmid p5-1ANW3u. The construct pT3HA5-1W3u that contained a heterologous 3'UTR was generated by replacing the ApaI-NotI DNA fragment of pT3HA5-1 with that of p51ANW3u (Fig. 1A).

Confirmation of hybrid viruses in infected plants. The in vitro transcription was carried out with individual NotI-linearized hybrid clones, and each transcription mixture was mechanically introduced into papaya and horn melon seedlings as described previously (6). The inoculated plants were kept in a temperaturecontrolled $\left(25\right.$ to $\left.28^{\circ} \mathrm{C}\right)$ greenhouse for observation of symptom development. The fidelity of the constructed hybrid viruses showing infectivity on horn melon were confirmed by reverse transcription-polymerase chain reaction (RT-PCR). The NruI site present at nucleotides 9958-9963 (in the CP coding region) and the RsaI site at 10174-10177 (in the 3'UTR) of HA5-1, but not present in W-CI (Fig. 1A), were used as markers. RT was performed with oligo-dT primer and Moloney murine leukemia virus (MMLV) reverse transcriptase (Promega). The DNA fragment from nucleotide positions 9860 to 10326 of PRSV was amplified with primers PW9860 (5'-ATGCCGCGGTATGGAATCAAG-3') and MW10326 (5'-CTCTCATTCCAAGAGGCTCG-3'), digested with NruI or RsaI, and analyzed by agarose (1.2\%) gel electrophoresis (Fig. 1B).

Cross-protection test. Chimeric mild viruses HA5-1Wcp3u, HA5-1Wcp, and HA5-1W3u (derived from infectious hybrid clones pT3HA5-1Wcp3u, pT3HA5-1Wcp, and pT3HA5-1W3u, respectively) did not cause conspicuous symptoms on plants of horn melon, zucchini squash, and papaya. The effectiveness of cross protection provided by these mild viruses against PRSV W$\mathrm{CI}$ and P-HA infections was evaluated under temperature-controlled $\left(25\right.$ to $\left.28^{\circ} \mathrm{C}\right)$ greenhouse conditions. Inocula of the mild strains were prepared by extracting 1 part of leaf tissue of horn melon with 20 parts of $0.01 \mathrm{M}$ phosphate buffer $(\mathrm{pH} 7.0$, wt/vol) at 15 days after inoculation. Horn melon plants at the stage of two true leaves and zucchini squash at two cotyledons were first inoculated with individual mild viruses or mock-inoculated with the buffer. Double-antibody sandwich enzyme-linked immunosorbent assay (DAS-ELISA) using the antiserum to PRSV (14) was performed to ensure the presence of the mild strains in the upper noninoculated leaves 2 weeks after inoculation. The challenge inoculation with severe viruses W-CI or P-HA was applied on two upper fully expanded leaves of horn melon or one of squash at 20 days after the mild strain inoculation. The inocula of severe viruses W-CI and P-HA were prepared, 15 days after inoculation, from leaves of virus-infected horn melon with $0.01 \mathrm{M}$ phosphate buffer ( $\mathrm{pH} 7.0)$ at 1:200 dilutions (wt/vol). Protection was recorded as plants showing only mild symptoms after the challenge inoculation at the time when the nonprotected controls developed severe symptoms. Breakdown of protection was defined by the appearance of severe mosaic symptoms. To evaluate the effectiveness of protection against W-CI infection, four experiments with a total of 40 plants of horn melon and three experiments with 30 plants of zucchini squash were conducted for each protective virus. For protection against P-HA, three experiments with a total of 30 plants of horn melon and two experiments with 20 plants of zucchini squash were conducted for each protective virus. Data were analyzed by independent samples $t$ test to determine the significance of difference $(P<0.05)$.

When the P type host papaya was used for assay, seedlings at the three- to four-leaf stage were inoculated with individual mild viruses or mock-inoculated with the buffer. Severe virus P-HA was used to inoculate two upper fully expanded leaves at 35 days after the mild strain inoculation. Three experiments with a total of 30 plants for each protective virus were conducted.

Determination of virus accumulation. Accumulation of recombinant mild viruses was determined at various times after inoculation. Two leaf disks of $6 \mathrm{~mm}$ were punched from each of three upper fully expanded leaves and one expanding young leaf of plants inoculated with individual mild viruses. DAS-ELISA (14) was used to determine virus accumulation. The absorbance $\left(A_{405}\right)$ was determined with an ELISA reader (Elx800, Bio-Tek Instrument, Inc., Winooski, VT) $1 \mathrm{~h}$ after the addition of substrate $\rho$-nitrophenyl phosphate $(1 \mathrm{mg} / \mathrm{ml})$. For each treatment, samples from eight plants were tested and the averages of $A_{405}$ with standard deviations were recorded. Independent samples $t$ test was performed to determine the significance of difference $(P<0.05)$.

Northern blot analysis. Samples for northern blot analysis were taken the same way as for ELISA from leaves of virus-infected papaya, horn melon, or zucchini plants. Total RNAs $(15 \mu \mathrm{g})$, extracted using the ULTRASPEC RNA isolation system (Biotecx Laboratories, Houston, TX), were separated on $1.2 \%$ agarose gel containing $0.66 \%$ formaldehyde, transferred to a Gene Screen Plus nylon membrane (DuPont Co., Boston, MA) using PosiBlot 30-30 (Stratagene, La Jolla, CA), and then hybridized with the ${ }^{32} \mathrm{P}$-labeled probe corresponding to cDNA fragment 6596-8891 of P-HA (47).

\section{RESULTS}

Verification of chimeric mild PRSV strains. The NruI and $R s a$ I sites present in HA5-1 (Fig. 1A), but not in W-CI, were used as makers to verify the fidelity of the recombinants. The DNA fragment of $101 \mathrm{bp}$ was released from PCR products of HA5-1 and HA5-1W3u after digestion with NruI. The DNA fragment of 150 bp was released from HA5-1 and HA5-1Wcp after digestion with RsaI. PCR products of W-CI and HA5-1Wcp3u were not digested with either enzyme (Fig. 1B). The results confirmed that, based on this restriction map, all the recombinants had the correct sequences as designed.

Enhancement of cross-protection effectiveness against W-CI by the CP coding region and $3^{\prime} \mathrm{UTR}$ of $\mathrm{W}$-CI. The recombinant HA5- 1 Wcp3u carrying the CP coding region and 3'UTR of W-CI and other chimeric mild strains infected horn melon (Fig. 2) and squash (data not shown) plants with no conspicuous symptoms, which was similar to that induced by HA5-1 (Fig. 2). The mockinoculated control of horn melon plants showed prominent mosaic and distortion on the upper leaves 7 to 9 days after inoculation with W-CI. A lack of protection was defined by the appearance of typical severe symptoms of mosaic and distortion on leaves of horn melon plants after challenge inoculation with W-CI (Fig. 2). Protection was recorded as plants showing no conspicuous symptoms after the challenge inoculation, similar to the plants inoculated with individual mild strains only (Fig. 2), at the time when the mock controls showed severe symptoms. Six weeks after W-CI challenge inoculation, $65 \%$ (26 out of 40 plants from four experiments) of HA5-1Wcp3u-inoculated horn melon plants were effectively protected without severe symptoms (Fig. $3 \mathrm{~A}$ ), whereas only $10 \%$ (4/40 plants) of HA5-1-inoculated plants did not show severe symptoms (Fig. 3A). There was a significant difference $(P<0.001)$ in protection effectiveness, indicating that HA5-1Wcp3u provided a much higher degree of protection against W-CI in horn melon than occurred for HA5-1.

The cross-protection effectiveness of HA5-1Wcp3u was also evaluated on zucchini squash (Fig. 3B). All plants of mock-inoculated squash showed severe symptoms of yellow spots and mosaic on the upper leaves 5 to 8 days after inoculation. HA5-1Wcp3u provided $100 \%$ (30/30 plants from three experiments) protection 
against $\mathrm{W}-\mathrm{CI}$ at 5 weeks after challenge inoculation and $97 \%$ protection at 6 weeks (29/30 plants). In contrast, severe symptoms developed on all plants protected with HA5-1 at 3 weeks after challenge inoculation with W-CI. The protection rates of HA5-1 and HA5-1Wcp3u were significantly different $(P<0.001)$ 6 weeks after the challenge inoculation. Thus, a much higher degree of protection against W-CI was also noticed in squash plants protected with HA5-1Wcp3u than those protected with HA5-1.

Enhancement of cross-protection effectiveness against W-CI by the 3'UTR of W-CI in horn melon. To investigate the roles of the heterologous $3^{\prime} \mathrm{UTR}$ and the $\mathrm{CP}$ coding region in cross protection against $\mathrm{W}-\mathrm{CI}$, recombinants HA5-1W3u carrying a heterologous 3'UTR and HA5-1Wcp carrying a heterologous CP coding region were evaluated, respectively. On horn melon plants, 6 weeks after challenge inoculation, HA5-1W3u provided a higher degree of protection $(58 \%, 23 / 40$ plants from four experiments) against W-CI than did HA5-1 (10\%, 4/40 plants) (Fig. 3A) and the difference was significant $(P=0.019)$. However, on squash plants the cross-protection effectiveness provided by HA5-1W3u against WCI (3\%, 1/30 plants from three experiments) was not significantly enhanced $(P=0.423)$ compared with that provided by HA5-1 $(0 \%, 0 / 30$ plants) (Fig. 3B). The results indicated that the heterologous 3'UTR of W-CI of the chimeric mild strain HA5-1W3u significantly increases cross-protection effectiveness against W-CI in horn melon, but not in squash.
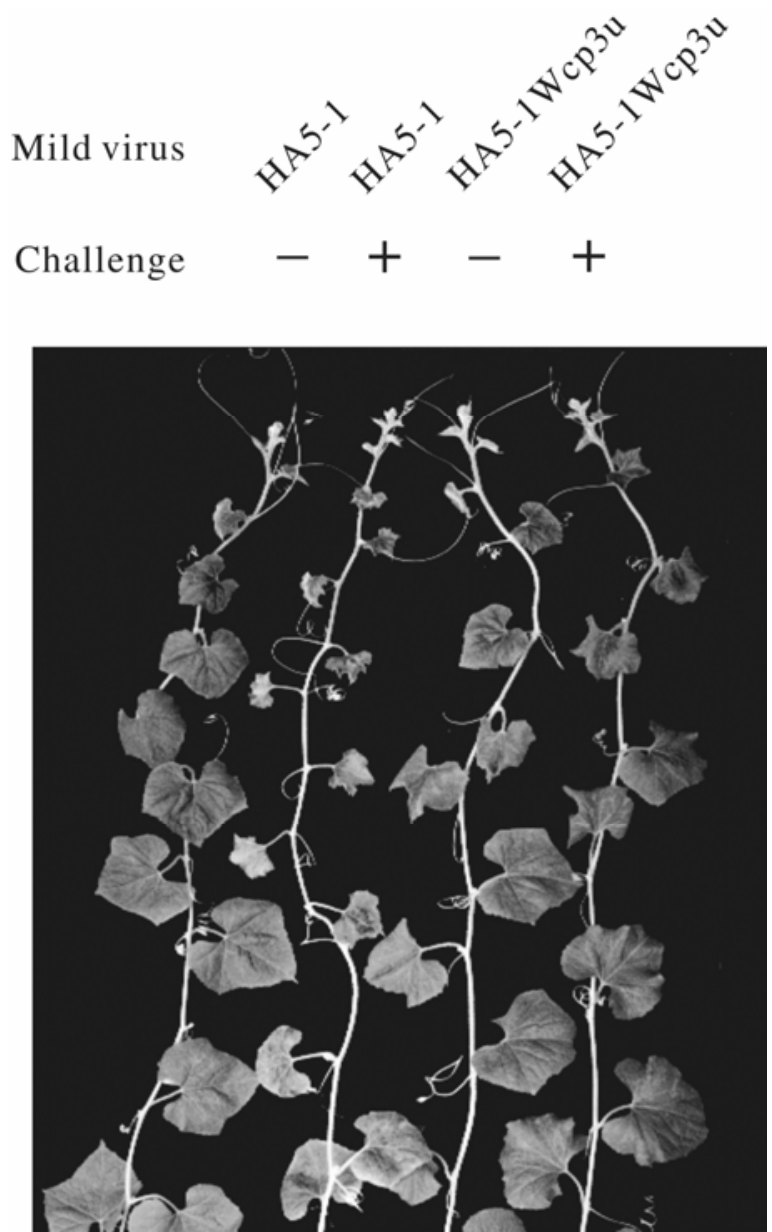

Fig. 2. Comparison of cross-protection effectiveness of the chimeric mild strain HA5-1Wcp3u in horn melon plants against Papaya ringspot virus (PRSV) type W virus with that provided by HA5-1. The plants were first mechanically inoculated with HA5-1 or HA5-1Wcp3u and then mechanically challenged with PRSV type W virus W-CI 20 days after the protective inoculation. Plants were photographed 20 days after challenge inoculation. + and represent the plants challenged with and without the severe strain W-CI, respectively.
In horn melon plants, HA5-1Wcp provided a similar degree of protection $(8 \%, 3 / 40$ plants from four experiments) as HA5-1 did (10\%) 6 weeks after challenge inoculation (Fig. 3A). In squash plants, the cross-protection effectiveness provided by HA5-1Wcp (7\%, 2/30 plants from three experiments) against W-CI was not significantly enhanced compared with that provided by HA5-1 (0\%) (Fig. 3B). The $P$ values were 0.62 and 0.184 for horn melon and squash, respectively. The results indicated that the heterologous CP coding region of W-CI alone did not significantly increase the effectiveness of cross protection of the recombinants against $\mathrm{W}-\mathrm{CI}$ in cucurbits.

Cross-protection effectiveness against the homologous $P$ type virus in cucurbits and papaya. P-HA induced prominent yellow mosaic, distortion, and narrowing on leaves of mockinoculated plants of horn melon 7 to 10 days after inoculation. Severe symptoms of mosaic with diffuse yellow spots and distortion were shown on leaves of mock-inoculated squash plants 10 to 18 days after P-HA inoculation. Seven weeks after the challenge inoculation, HA5-1 and all the chimeric mild viruses showed $100 \%$ (30/30 from three experiments) protection against P-HA in horn melon (Fig. 4A). Complete protection against P-HA was also observed with HA5-1Wcp3u and HA5-1Wcp on zucchini squash and $90 \%$ (18/20 plants from two experiments) of squash plants inoculated with HA5-1 or HA5-1W3u were protected (Fig. 4B). All chimeric mild strains maintained high degrees of protection against P-HA infection in cucurbits up to 2 months (data not shown).

In papaya, all mock-inoculated plants showed prominent vein clearing on young leaves 10 to 18 days after P-HA inoculation
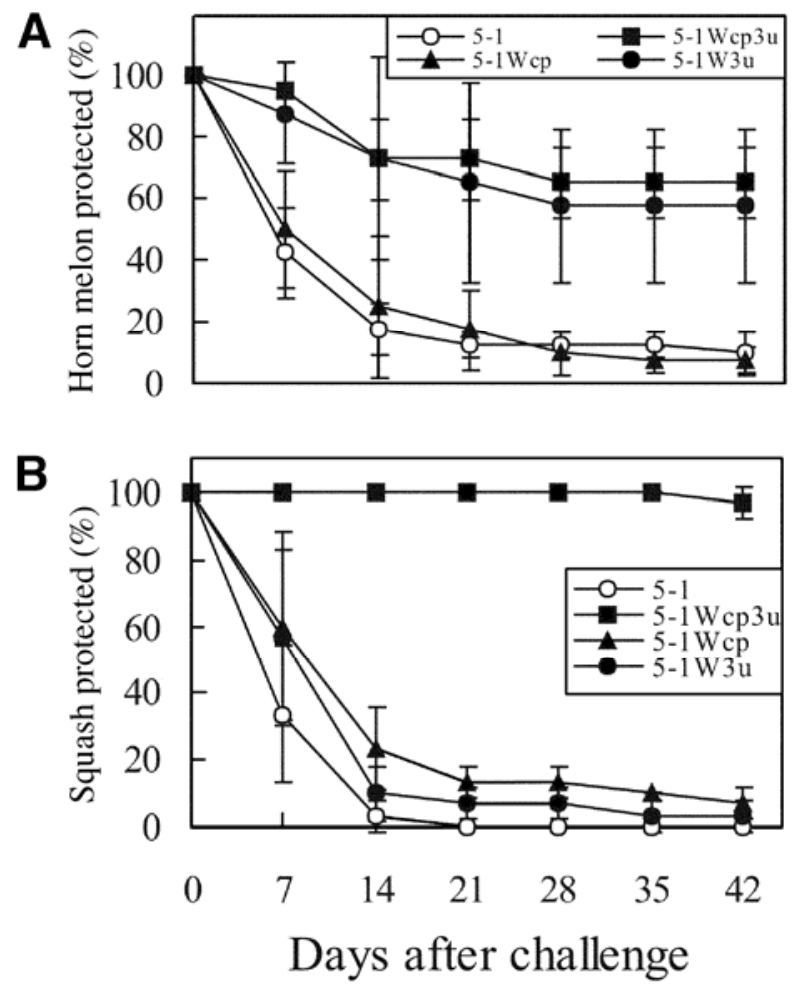

Fig. 3. Cross-protection effectiveness provided by the type P mild strain HA51 of Papaya ringspot virus (PRSV) and its various recombinants against PRSV type W virus W-CI on plants of $\mathbf{A}$, horn melon and $\mathbf{B}$, zucchini squash. Test plants were first inoculated with individual viruses of HA5-1 (5-1), HA51Wcp3u (5-1Wcp3u), HA5-1Wcp (5-1Wcp), and HA5-1W3u (5-1W3u) and then challenged with W-CI at 20 days after protective inoculation. Protection was defined as those plants showing only mild symptoms when the nonprotected controls showed severe symptoms. The data were combined from four experiments with a total of 40 plants tested for each treatment on horn melon and three experiments with 30 plants of zucchini squash. The narrow vertical bars represent standard deviation. 
and subsequently with severe yellow mosaic and distortion. Nine weeks after the challenge inoculation, $100 \%$ (30/30 plants from three experiments) and $87 \%$ (26/30 plants) of plants inoculated with HA5-1 and HA5-1Wcp3u, respectively, were protected from P-HA infection. However, only 40\% (12/30 plants) and 43\% (13/30 plants) of plants inoculated with HA5-1Wcp and HA5$1 \mathrm{~W} 3 \mathrm{u}$, respectively, were protected (Fig. 4C). Statistical analyses indicated that the chimeric mild strains containing a heterologous $\mathrm{CP}$ coding region (HA5-1Wcp) $(P=0.007)$ or a heterologous 3'UTR (HA5-1W3u) $(P=0.003)$ significantly reduced the cross-

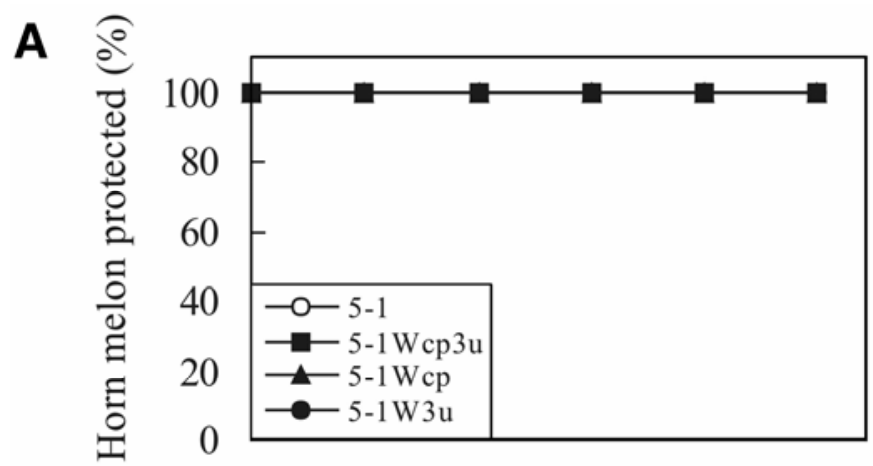

B

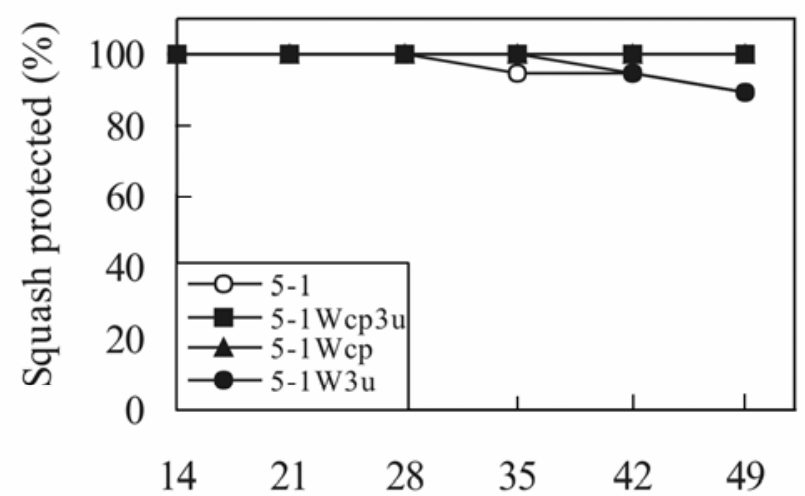

C

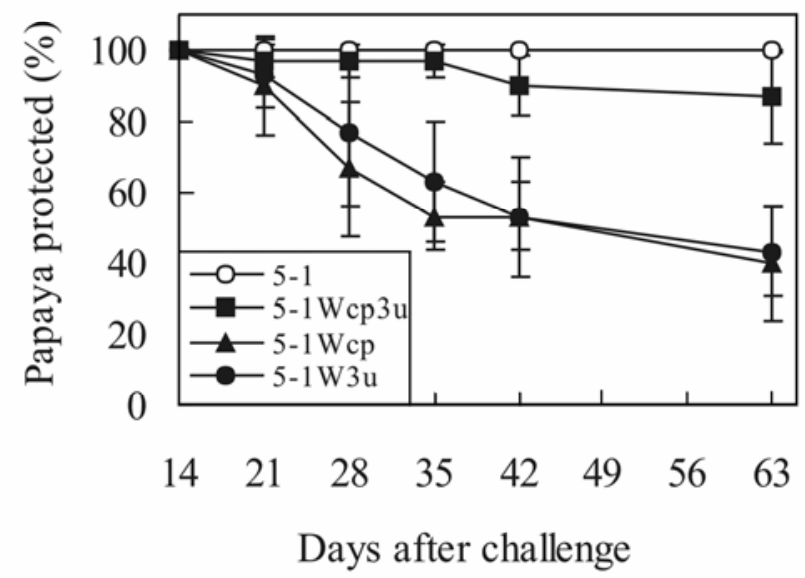

Fig. 4. Cross-protection effectiveness provided by various recombinants derived from mild strain HA5-1 on plants of A, horn melon, $\mathbf{B}$, zucchini squash, and $\mathbf{C}$, papaya after challenge with type $\mathbf{P}$ virus P-HA. Plants of horn melon, zucchini squash, and papaya were first inoculated with HA5-1 (5-1), HA5$1 \mathrm{Wcp} 3 \mathrm{u}(5-1 \mathrm{Wcp} 3 \mathrm{u})$, HA5-1Wcp (5-1Wcp), or HA5-1W3u (5-1W3u) and then challenged with P-HA at 20 (horn melon and zucchini squash) and 35 days (papaya) after protective inoculation. Breakdown of protection was defined by appearance of severe symptoms after challenge inoculation. The data were combined from two experiments with a total of 20 plants of zucchini squash and three experiments with 30 plants of horn melon and 30 plants of papaya tested. The narrow vertical bars represent standard deviation. protection effectiveness against type $\mathrm{P}$ virus $\mathrm{P}-\mathrm{HA}$ in papaya plants compared with those provided by HA5-1, but recombinant HA5-1Wcp3u containing both heterologous $\mathrm{CP}$ coding region and $3^{\prime}$ UTR did not $(P=0.205)$.

Virus accumulation of HA5-1 and the mild recombinants in protected plants. Virus accumulations of various mild strains in horn melon plants determined by ELISA were similar for HA5-1, HA5-1Wcp3u, and HA5-1W3u at 15 days after inoculation, but a lower $A_{405}$ value was obtained for HA5-1Wcp (Fig. 5A). Statistical analyses indicated that virus accumulation of HA5-1 was sig-
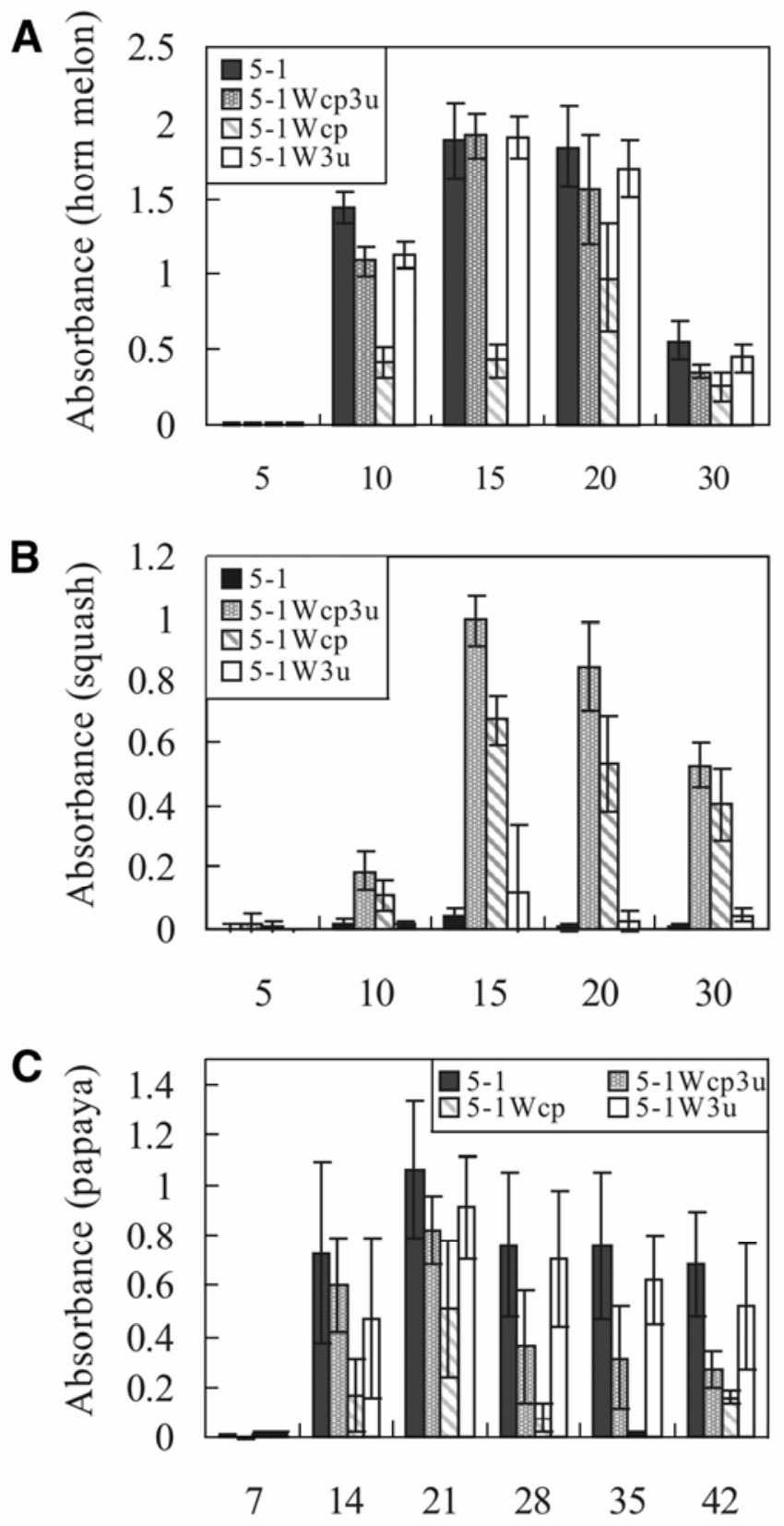

\section{Days after inoculation}

Fig. 5. Accumulation of viral coat protein of mild strain HA5-1 and its recombinants in $\mathbf{A}$, horn melon, $\mathbf{B}$, zucchini squash, and $\mathbf{C}$, papaya. Plants were inoculated with individual viruses of HA5-1 (5-1), HA5-1Wcp3u (5-1Wcp3u), HA5-1Wcp (5-1Wcp), or HA5-1W3u (5-1W3u). Double-antibody sandwich enzyme-linked immunosorbent assay (ELISA) using the antiserum to Papaya ringspot virus $\mathrm{HA}$ was performed to determine the accumulation of viral coat protein. Leaf disks individually punched from eight plants of each treatment after the mild strain inoculation were used for ELISA. The averages of $A_{405}$ with standard deviations are shown. The narrow vertical bars represent standard deviation. 
nificantly higher than HA5-1Wcp $(P<0.001)$, but similar to that of HA5-1Wcp3u $(P=0.746)$ and HA5-1W3u $(P=0.834)$. In squash plants, the accumulations of HA5-1Wcp3u and HA5$1 \mathrm{Wcp}$ were higher than those of HA5-1 and HA5-1W3u at 15 days after inoculation (Fig. 5B). Virus accumulation of HA5-1 was similar to HA5-1W3u $(P=0.383)$ and significantly lower than that of HA5-1Wcp3u $(P<0.001)$ and HA5-1Wcp $(P<$ $0.001)$. The virus accumulation of all mild strains reached a maximum at 15 days after inoculation in horn melon and squash plants (Fig. 5A and B). In papaya plants, the level of virus accumulation of HA5-1Wcp at 21 days after inoculation was the lowest compared with those of HA5-1, HA5-1Wcp3u, and HA5-1W3u (Fig. 5C). Virus accumulation of HA5-1 was significantly higher than HA5-1Wcp $(P=0.002)$ and similar to that of HA5-1Wcp3u $(P=$ $0.54)$ and HA5-1W3u $(P=0.263)$. The highest $A_{405}$ of all mild strains in papaya was noticed at 21 days after inoculation (Fig. $5 \mathrm{C})$. The relatively higher levels of virus accumulation of HA5-1, HA5-1Wcp $3 u$, and HA5-1W3u in horn melon, HA5-1Wcp3u in zucchini squash, and HA5-1 and HA5-1W3u in papaya were maintained in the same trends at different time intervals after inoculation (Fig. 5A to C).

The levels of viral RNA accumulation of the chimeric mild strains in plants were closely correlated with the levels of the accumulation of CP as determined by DAS-ELISA. Similar results of a positive correlation between the accumulation of viral RNA and that of CP were noticed at different time intervals after the inoculation of mild viruses (data not shown).

\section{DISCUSSION}

Similar to previous investigations $(43,44)$, the type P mild strain PRSV HA5-1 provided high degrees of protection against type $\mathrm{P}$ virus $\mathrm{P}-\mathrm{HA}$, but not against type $\mathrm{W}$ virus $\mathrm{W}-\mathrm{CI}$ in cucurbits. The chimeric mild virus HA5-1Wcp3u carrying the heterologous $\mathrm{CP}$ coding region and the $3^{\prime} \mathrm{UTR}$ from W-CI provided a much higher degree of cross protection against the type W virus W-CI than HA5-1 did (Fig. 3A and B), while still maintaining a high degree of cross protection against the type $\mathrm{P}$ virus $\mathrm{P}-\mathrm{HA}$ in cucurbits (Fig. 4A and B). Our results indicate that it is possible to confer broad protection against two particular virus strains by a chimeric mild potyvirus carrying a heterologous $3^{\prime}$ genomic region. Since monoclonal antibody is not available and northern blotting is not useful to distinguish P-HA and W-CI, the breakdown of cross protection in this study was defined by the appearance of severe mosaic symptoms, which could be easily distinguished from the inconspicuous symptoms induced by the mild strains, and the concentrations of the challenge virus were not monitored.

A practically useful mild strain should not cause adverse effects to hosts and should maintain high stability without severe revertants arising (44). Through the recombination analyses between HA and HA5-1 at the cDNA level, the mutations affecting the pathogenicity of PRSV HA5-1 have been located in the P1, helper component-proteinase (HC-Pro), and P3 regions (5). The chimeric mild strains with the heterologous $\mathrm{CP}$ coding region and/or the 3'UTR from the severe PRSV strain W-CI did not show conspicuous symptoms in cucurbits and papaya. No severe revertants of the chimeric mild strains occurred after more than 1 year of passages (transferred once every month) in cucurbits (data not shown). The attenuation and stability of our chimeric mild strains make them suitable for control of PRSV type W viruses in cucurbits. The conserved DAG triplet of potyviral CP genes $(2,13,18)$ and the KITC motif of potyviral HC-Pro genes $(1,15)$ are essential for aphid transmissibility. To prevent the spread of the chimeric mild viruses in the field, the DAG motif in the $\mathrm{CP}$ coding region and the KITC motif in the HC-Pro coding region are currently being mutated to abolish their aphid transmissibility.

We also noticed that a heterologous $3^{\prime}$ UTR in a chimeric mild strain greatly affects the cross-protection effectiveness in particu- lar hosts. The 3'UTR of a potyvirus is involved in replication for positive-strand viral RNA (11). The RNA-dependent RNA polymerase (RdRp) complex, which consists of viral replicase $(4,19)$ and host factor(s) $(20,30)$, binds to the $3^{\prime}$ UTR of potyviral genome to initiate replication. We infer that through evolution, PRSV type W strains are more adapted to cucurbits than P strains and the genomic 3'UTRs of PRSV type W strains have stronger affinity for the RdRp complex than those of PRSV type P strains in cucurbits. A higher level of accumulation of the protective strain should have a stronger ability to compete for the aforementioned factors required for replication of the challenge virus. The fact that HA5-1W3u accumulated to higher levels (Fig. 5A) and provided higher degrees of protection against W-CI in horn melon (Fig. 3A) and HA5-1W3u accumulated at a fairly low level (Fig. $5 \mathrm{~B})$ and provided low degrees of protection against W-CI in squash (Fig. 3B) indicate that host factors play important roles for cross protection.

Preventing the uncoating of the challenge virus by protein interference of the $\mathrm{CP}$ of the protective virus has been proposed as a mechanism for cross protection $(10,35)$ and for CP gene transgenic resistance $(3,24,27,42)$. CP was proven to play an important role in cross protection by the approach of chimeric viruses. Chimeric PVX carrying a translatable TMV CP gene provides a better degree of protection against TMV than the chimeric PVX carrying an untranslatable $\mathrm{TMV} \mathrm{CP}$ gene, indicating that $\mathrm{CP}$ plays a more important role in cross protection than the nucleotide sequence (9). An amino acid substitution at the $\mathrm{N}$-terminal region of the CP of PVA tobacco strain reduced virus accumulation and the ability to overcome cross-protection provided by the potato strain (37). However, several lines of evidence pointed out that CP interference is not involved in cross protection. The defective mutant of TMV which produces insoluble defective CP (48) or CP-free TMV (34) was found to interfere with the challenge strains of TMV. Our results showed that the expression of type W CP by HA5-1Wcp did not provide a significantly higher degree of protection against $\mathrm{W}-\mathrm{CI}$ in horn melon and zucchini squash than that provided by HA5-1 (Fig. 3A and B), even though the $\mathrm{CP}$ accumulation of HA5-1Wcp is much higher than that of HA5-1 in squash (Fig. 5B). Our case from squash suggests that functional interference of $\mathrm{CP}$ at the protein level may not play an essential role for cross protection between PRSV P and W strains.

Sequence homology-dependent PTGS is considered an antiviral defense mechanism in virus-infected plants, playing an important role for cross protection (38). A higher degree of sequence identity between a protective strain and a challenge strain may increase the cross-protection effectiveness. HA5-1Wcp3u provided much higher degrees of protection against W-CI in horn melon and squash plants than HA5-1 did (Fig. 3A and B). This may be the result from the identical sequences of the $\mathrm{CP}$ region and 3'UTR between HA5-1Wcp3u and W-CI through a PTGS mechanism. High degrees of protection provided by all chimeric mild strains against P-HA in horn melon and squash plants (Fig. 4A and B) may be triggered by PTGS induced by the long homologous RNA segments shared by the individual chimeric mild strains and the strain P-HA. However, some of our results cannot be solely explained by the PTGS mechanism. The fact that the heterologous CP coding region and the 3'UTR of the chimeric mild strains HA5-1Wcp and HA5-1W3u, respectively, greatly reduced their cross-protection effectiveness against P-HA in papaya plants (Fig. 4C) and HA5-1Wcp3u maintained high degrees of protection against P-HA (Fig. 4C) indicate that the protection effectiveness is not positively correlated with sequence identity between mild and severe challenge strains. Previous studies showed that the secondary structure of the C-terminal half of the CP coding region and the 3'UTR of Tobacco etch virus are important for genome amplification $(16,25)$. The heterologous $\mathrm{CP}$ coding region and the $3^{\prime} \mathrm{UTR}$ of HA5-1Wcp and HA5-1W3u, respectively, may affect the RNA secondary structure of the $3^{\prime}$ genomic region, 
thus reducing the interaction strength with the RdRp complex. On the other hand, the $3^{\prime}$ genomic region of HA5-1Wcp3u containing both heterologous $\mathrm{CP}$ coding region and 3'UTR may possess a more fitting structure to restore the competitive ability and provided a high degree of protection against $\mathrm{P}-\mathrm{HA}$ similar to that provided by HA5-1 (Fig. 4C). However, the reduction of protection against $\mathrm{P}-\mathrm{HA}$ by the heterologous $\mathrm{CP}$ coding region or the 3'UTR from W-CI was only shown in papaya (Fig. 4C), not in cucurbits (Fig. 4A and B). These results imply that the effects of the heterologous $\mathrm{CP}$ coding region and the $3^{\prime} \mathrm{UTR}$ on cross protection are host dependent.

In conclusion, we show that our chimeric attenuated virus strains have a good potential for control of $\mathrm{P}$ and $\mathrm{W}$ type viruses of PRSV in cucurbits. The organization of the 3' viral genomic region, virus accumulation, and host factors affected the crossprotection effectiveness of the chimeric recombinants. In addition, the importance of the other viral genes or genomic regions required for cross protection can be further identified by our recombination system.

\section{ACKNOWLEDGMENTS}

This project was supported by the grants (NSC 89-2321-B-005-001 and NSC 90-2321-B-005-001) from National Science Council of the Republic of China on Taiwan.

\section{LITERATURE CITED}

1. Atreya, C. D., Atreya, P. L., Thornbury, D. W., and Pirone, T. P. 1992. Site-directed mutations in the potyvirus HC-Pro gene affect helper component activity, virus accumulation, and symptom expression in infected tobacco plants. Virology 191:106-111.

2. Atreya, C. D., Raccah, B., and Pirone, T. P. 1990. A point mutation in the coat protein abolishes aphid transmissibility of a potyvirus. Virology 178:161-165.

3. Bendahmane, M., Fitchen, J. H., Zhang, G., and Beachy, R. N. 1997. Studies of coat protein-mediated resistance to tobacco mosaic tobamovirus: Correlation between assembly of mutant coat proteins and resistance. J. Virol. 71:7942-7950.

4. Buck, K. W. 1996. Comparison of the replication of positive-stranded RNA viruses of plants and animals. Pages 159-251 in: Advances in Virus Research. K. Maramorosch, F. A. Murphy, and A. J. Shatkin, eds. Academic Press, San Diego, CA.

5. Chiang, C. H. 1995. Construction of in vitro and in vivo infectious transcripts of papaya ringspot potyvirus and analysis on pathogenicity of virus hybrids. Ph.D. thesis. National Chung Hsing University, Taichung, Taiwan.

6. Chiang, C. H., and Yeh, S. D. 1997. Infectivity assays of in vitro and in vivo transcripts of papaya ringspot potyvirus. Bot. Bull. Acad. Sinica 38:153-163.

7. Costa, A. S., and Muller, G. W. 1980. Tristeza control by cross protection: A U.S.-Brazil cooperative success. Plant Dis. 64:538-541.

8. Covey, S. N., Al-Kaff, N. S., Langara, A., and Turner, D. S. 1997. Plants combat infection by gene silencing. Nature 385:781-782.

9. Culver, J. N. 1996. Tobamovirus cross protection using a potexvirus vector. Virology 226:228-235.

10. Dodds, J. A. 1982. Cross-protection and interference between electrophoretically distinct strains of cucumber mosaic virus in tomato. Virology 118:235-240.

11. Dreher, T. W. 1999. Functions of the 3'-untranslated regions of positive strand RNA viral genomes. Annu. Rev. Phytopathol. 37:151-174.

12. Fulton, R. W. 1978. Superinfection by strains of tobacco streak virus. Virology 85:1-8.

13. Gal-On, A., Antignus, Y., Rosner, A., and Raccah, B. 1992. A zucchini yellow mosaic virus coat protein gene mutation restores aphid transmissibility but has no effect on multiplication. J. Gen. Virol. 73:21832187.

14. Gonsalves, D., and Ishii, M. 1980. Purification and serology of papaya ringspot virus. Phytopathology 70:1028-1032.

15. Granier, F., Durand-Tardif, M., Casse-Delbart, F., Lecoq, H., and Robaglia, C. 1993. Mutations in zucchini yellow mosaic virus helper component protein associated with loss of aphid transmissibility. J. Gen. Virol. 74:2737-2742.

16. Haldeman-Cahill, R., Daros, J. A., and Carrington, J. C. 1998. Secondary structures in the capsid protein coding sequence and $3^{\prime}$ nontranslated region involved in amplification of the tobacco etch virus genome. J. Virol. 72:4072-4079.

17. Hamilton, A. J., and Baulcombe, D. C. 1999. A species of small antisense RNA in posttranscriptional gene silencing in plants. Science 286:950-952.

18. Harrison, B. D., and Robinson, D. J. 1988. Molecular variation in vectorborne plant viruses: Epidemiological significance. Philos. Trans. R. Soc. Lond. B Biol. Sci. 321:447-462.

19. Ishihama, A., and Barbier, P. 1994. Molecular anatomy of viral RNAdirected RNA polymerases. Arch. Virol. 134:235-258.

20. Ishikawa, M., Diez, J., Restrepo-Hartwig, M., and Ahlquist, P. 1997. Yeast mutations in multiple complementation groups inhibit brome mosaic virus RNA replication and transcription and perturb regulated expression of the viral polymerase-like gene. Proc. Natl. Acad. Sci. USA 94:13810-13815.

21. Krstic, B., Ford, R. E., Shukla, D. D., and Tosic, M. 1995. Cross-protection studies between strains of sugarcane mosaic, maize dwarf mosaic, Johnsongrass mosaic, and sorghum mosaic potyviruses. Plant Dis. 79:135-138.

22. Lecoq, H., Lemaire, J. M., and Wipf-Scheibel, C. 1991. Control of zucchini yellow mosaic virus in squash by cross protection. Plant Dis. 75:208-211.

23. Lindbo, J. A., Silva-Rosales, L., Proebsting, W. M., and Dougherty, W. G. 1993. Induction of a highly specific antiviral state in transgenic plants: Implications for regulation of gene expression and virus resistance. Plant Cell 5:1749-1759.

24. Lu, B., Stubbs, G., and Culver, J. N. 1998. Coat protein interactions involved in tobacco mosaic tobamovirus cross-protection. Virology 248:188-198.

25. Mahajan, S., Dolja, V. V., and Carrington, J. C. 1996. Roles of the sequence encoding tobacco etch virus capsid protein in genome amplification: Requirements for the translation process and a cis-active element. J. Virol. 70:4370-4379.

26. Mckinney, H. H. 1929. Mosaic diseases in the Canary Islands, West Africa, and Gibraltar. J. Agric. Res. 39:557-578.

27. Osbourn, J. K., Watts, J. W., Beachy, R. N., and Wilson, T. M. 1989. Evidence that nucleocapsid disassembly and a later step in virus replication are inhibited in transgenic tobacco protoplasts expressing TMV coat protein. Virology 172:370-373.

28. Ponz, F., and Bruening, G. 1986. Mechanisms of resistance to plant viruses. Annu. Rev. Phytopathol. 24:355-381.

29. Purcifull, D. E., Edwardson, J., Hiebert, E., and Gonsalves, D. 1984. Papaya ringspot virus. No. 292 in: Descriptions of Plant Viruses. Commonw. Mycol. Inst./Assoc. Appl. Biol., Kew, England.

30. Quadt, R., Kao, C. C., Browning, K. S., Hershberger, R. P., and Ahlquist, P. 1993. Characterization of a host protein associated with brome mosaic virus RNA-dependent RNA polymerase. Proc. Natl. Acad. Sci. USA 90:1498-1502.

31. Rast, A. T. B. 1972. MII-16, an artificial symptomless mutant of tobacco mosaic virus for seedling inoculation of tomato crops. Neth. J. Plant Pathol. 78:110-112.

32. Ratcliff, F., Harrison, B. D., and Baulcombe, D. C. 1997. A similarity between viral defense and gene silencing in plants. Science 276:15581560.

33. Ratcliff, F. G., MacFarlane, S. A., and Baulcombe, D. C. 1999. Gene silencing without DNA: RNA-mediated cross-protection between viruses. Plant Cell 11:1207-1215.

34. Sarkar, S., and Smitamana, P. 1981. A proteinless mutant of tobacco mosaic virus: Evidence against the role of a viral coat protein for interference. Mol. Gen. Genet. 184:158-159.

35. Sherwood, J. L., and Fulton, R. W. 1982. The specific involvement of coat protein in tobacco mosaic virus cross protection. Virology 119:150-158.

36. Smith, H. A., Swaney, S. L., Parks, T. D., Wernsman, E. A., and Dougherty, W. G. 1994. Transgenic plant virus resistance mediated by untranslatable sense RNAs: Expression, regulation, and fate of nonessential RNAs. Plant Cell 6:1441-1453.

37. Valkonen, J. P. T., Rajamaki, M. L., and Kekarainen, T. 2002. Mapping of viral genomic regions important in cross-protection between strains of a potyvirus. Mol. Plant-Microbe Interact. 15:683-692.

38. Voinnet, O. 2001. RNA silencing as a plant immune system against viruses. Trends Genet. 17:449-459.

39. Wang, H. L., Gonsalves, D., Provvidenti, R., and Lecoq, H. L. 1991. Effectiveness of cross protection by a mild strain of zucchini yellow mosaic virus in cucumber melon and squash. Plant Dis. 75:203-207.

40. Wang, J. J., and Yeh, S. D. 1998. Characterization of the papaya ringspot virus $\mathrm{W}$ type isolates collected from different areas of Taiwan by host reactions, immunodiffusion tests and RT-PCR. Plant Prot. Bull. 40:383395.

41. Wen, F., Lister, R. M., and Fattouh, F. A. 1991. Cross-protection among strains of barley yellow dwarf virus. J. Gen. Virol. 72:791-799.

42. Wu, X. J., Beachy, R. N., Wilson, T. M., and Shaw, J. G. 1990. Inhibition of uncoating of tobacco mosaic virus particles in protoplasts from trans- 
genic tobacco plants that express the viral coat protein gene. Virology 179:893-895.

43. Yeh, S. D., and Gonsalves, D. 1984. Evaluation of induced mutants of papaya ringspot virus for control by cross protection. Phytopathology 74:1086-1091.

44. Yeh, S. D., and Gonsalves, D. 1994. Practices and perspective of control of papaya ringspot virus by cross protection. Pages 237-257 in: Advances in Disease Vector Research. K. F. Harris, ed. Springer-Verlag, New York.

45. Yeh, S. D., Gonsalves, D., and Provvidenti, R. 1984. Comparative studies on host range and serology of papaya ringspot virus and watermelon mosaic virus 1. Phytopathology 74:1081-1085.

46. Yeh, S. D., Gonsalves, D., Wang, H. L., Namba, R., and Chiu, R. J. 1988. Control of papaya ringspot virus by cross protection. Plant Dis. 72:375380 .

47. Yeh, S. D., Jan, F. J., Chiang, C. H., Doong, T. J., Chen, M. C., Chung, P. H., and Bau, H. J. 1992. Complete nucleotide sequence and genetic organization of papaya ringspot virus RNA. J. Gen. Virol. 73:2531-2541.

48. Zaitlin, M. 1976. Viral cross-protection: More understanding is needed. Phytopathology 66:382-383. 